\title{
Spectral Minutiae: A Fixed-length Representation of a Minutiae Set
}

\author{
Haiyun Xu, Raymond N.J. Veldhuis \\ University of Twente, Department of Electrical Engineering \\ P.O. box 217, 7500 AE Enschede, The Netherlands \\ $\{\mathrm{h} . \mathrm{xu}, \mathrm{r} \cdot \mathrm{n} \cdot \mathrm{j} \cdot \mathrm{vel}$ dhuis\}@el.utwente.nl \\ Tom A.M. Kevenaar, Anton H.M. Akkermans \\ Philips Research Laboratories \\ Prof. Holstlaan 4, 5656 AA Eindhoven, The Netherlands \\ \{tom.kevenaar, ton.h.akkermans\}@philips.nl \\ Asker M. Bazen \\ Uniqkey Biometrics \\ Graafvorkhoek 22, 7546 KK Enschede, The Netherlands \\ a.m.bazen@uniqkey.com
}

\begin{abstract}
Minutiae, which are the endpoints and bifurcations of fingerprint ridges, allow a very discriminative classification of fingerprints. However, a minutiae set is an unordered set and the minutiae locations suffer from various deformations such as translation, rotation and scaling. In this paper, we introduce a novel method to represent a minutiae set as a fixed-length feature vector, which is invariant to translation, and in which rotation and scaling become translations, so that they can be easily compensated for. By applying the spectral minutiae representation, we can combine the fingerprint recognition system with a template protection scheme, which requires a fixed-length feature vector. This paper also presents two spectral minutiae matching algorithms and shows experimental results.
\end{abstract}

\section{Introduction}

A fingerprint consists of a pattern of line structures, which are called ridges. The most prominent ridge characteristics are minutiae, which are the ridge endpoints and bifurcations. They are known to remain unchanged over an individual's lifetime [11]. Minutiae-based fingerprint recognition techniques are popular and widely used [5, 9]. However, they have some drawbacks, which limit their application. First, due to the fact that minutiae sets are unordered, the correspondence between individual minutia in two minutiae sets is unknown before matching and this makes it difficult to find the geometric transformation (consisting of translation, rotation, scaling, and optionally nonlinear deformations [5]) that optimally registers (or aligns) two sets. For fingerprint identification systems with very large databases [2], in which a fast comparison algorithm is necessary, minutiae-based matching algorithms will fail to meet the high speed requirements. Secondly, a minutiae representation of a fingerprint cannot be applied directly in recently developed template protection schemes $[15,16]$, which require as an input a fixed-length feature vector representation of a biometric modality. The spectral minutiae representation as proposed in this paper overcomes the above drawbacks of the minutiae sets, thus broadening the application of minutiae-based algorithms.

There are several algorithms to extract a fixed-length feature vector from fingerprints. The FingerCode as presented in [10] is based on ridge features. The author concluded that FingerCodes are not as distinctive as minutiae and they can be used as complementary information for fingerprint matching. Willis and Myers brought forward a fixed-length minutiae wedge-ring feature [18], which recorded the minutiae numbers on a pattern of wedges and rings. However, this method can only perform a coarse fingerprint authentication, and cannot handle big translations and rotations. Recently, a feature vector based on the distribution of the pairwise distances between minutiae is proposed by Park $e t$ al. [13]. However, this algorithm is only evaluated on the manually labelled minutiae and the performance is not satisfying.

Our method is inspired by the Fourier-Mellin transform, 
which was first introduced by the optical research community [7]. It was often used in image processing to obtain a translation, rotation and scaling invariant descriptor of the image $[8,14]$. However, the implementation of the Fourier-Mellin transform requires a Fourier transform and a polar-logarithmic mapping. When applying those on a digital image, a resampling and interpolation process is normally unavoidable. To avoid the interpolation errors, we introduce an analytical representation of the minutiae set, and then use analytical expressions of a continuous Fourier transform that can be evaluated on polar-logarithmic coordinates. By representing minutiae in the spectral domain, we transform a minutiae set into a fixed-length feature vector, which at the same time does not need registration to compensate for translation, rotation and scaling. By using a spectral minutiae representation instead of minutiae sets, we meet the requirements of template protection and allow for faster matching as well.

The spectral minutiae representation method can be easily integrated into a minutiae-based fingerprint recognition system. Minutiae sets can be directly transformed to this new representation, which makes this method compatible with the large amount of existing minutiae databases.

This paper is organized as follows. First, in Section 2, the concept of spectral minutiae representation is explained in detail. Next, two correlation-based spectral minutiae matching algorithms are proposed in Section 3. Then, Section 4 will present the experimental results. Finally, we will draw conclusions in Section 5.

\section{Spectral Minutiae Representation}

\subsection{Background}

The spectral minutiae representation is based on the shift, scale and rotation properties of the two-dimensional continuous Fourier transform. If we have an input signal $f(\vec{x}), \vec{x}=(x, y)^{\mathrm{T}}$ (we denote the transpose of a vector $\vec{v}$ as $\vec{v}^{\mathrm{T}}$ ), its continuous Fourier transform is

$$
\mathscr{F}\{f(\vec{x})\}=F(\vec{\omega})=\int_{-\infty}^{\infty} \int_{-\infty}^{\infty} f(\vec{x}) \exp \left(-\mathrm{j} \vec{w}^{\mathrm{T}} \vec{x}\right) \mathrm{d} \vec{x},
$$

with $\vec{\omega}=\left(\omega_{x}, \omega_{y}\right)^{\mathrm{T}}$. The Fourier transform of a translated $f(\vec{x})$ is

$$
\mathscr{F}\left\{f\left(\vec{x}-\vec{x}_{0}\right)\right\}=\exp \left(-\mathrm{j} \vec{\omega}^{\mathrm{T}} \vec{x}_{0}\right) F(\vec{\omega}),
$$

with $\vec{x}_{0}=\left(x_{0}, y_{0}\right)^{\mathrm{T}}$ the translation vector. The Fourier transform of an isotropically scaled $f(\vec{x})$ is

$$
\mathscr{F}\{f(a \vec{x})\}=a^{-2} F\left(a^{-1} \vec{\omega}\right),
$$

with $a(a>0)$ the isotropic scaling factor. The Fourier transform of a rotated $f(\vec{x})$ is

$$
\mathscr{F}\{f(\Phi \vec{x})\}=F(\Phi \vec{\omega})
$$

with

$$
\Phi=\left(\begin{array}{cc}
\cos \phi & -\sin \phi \\
\sin \phi & \cos \phi
\end{array}\right)
$$

Here $\Phi$ is the (orthonormal) rotation matrix and $\phi$ is the (anticlockwise) rotation angle of $f(\vec{x})$.

It can be seen from (2) that if only the magnitude of the Fourier spectrum is retained, this results in a translation invariant representation of the input signal. Furthermore, from (3) and (4) it follows that scaling and rotation of the input signal results in a scaled and rotated Fourier spectrum.

Based on the above properties of the two-dimensional Fourier transform, we can re-map the Fourier spectral magnitude onto a polar-logarithmic coordinate system with respect to an origin, such that the rotation and scaling become translations along the angular and radial axes, respectively. The detailed steps are as follows. Consider a signal $t(\vec{x})$ that is translated, scaled and rotated replica of $r(\vec{x})$,

$$
t(\vec{x})=r\left(a \Phi \vec{x}-\vec{x}_{0}\right),
$$

then the magnitude of the Fourier transforms of $t(\vec{x})$ and $r(\vec{x})$ are related by,

$$
|T(\vec{\omega})|=a^{-2}\left|R\left(a^{-1} \Phi \vec{\omega}\right)\right|,
$$

which is a translation invariant representation of the input signal. If we re-map the Fourier spectral magnitude onto a polar-logarithmic coordinate system as,

$$
\begin{gathered}
\lambda=\log \sqrt{\omega_{\mathrm{x}}^{2}+\omega_{\mathrm{y}}^{2}}, \quad \beta=\arctan \left(\frac{\omega_{\mathrm{y}}}{\omega_{\mathrm{x}}}\right), \\
R_{\mathrm{pl}}(\lambda, \beta)=\left|R\left(\mathrm{e}^{\lambda} \cos \beta, \mathrm{e}^{\lambda} \sin \beta\right)\right|, \\
T_{\mathrm{pl}}(\lambda, \beta)=\left|T\left(\mathrm{e}^{\lambda} \cos \beta, \mathrm{e}^{\lambda} \sin \beta\right)\right|,
\end{gathered}
$$

then we have the Fourier spectral magnitude of $t(\vec{x})$ and $r(\vec{x})$ on the polar-logarithmic coordinates,

$$
T_{\mathrm{pl}}(\lambda, \beta)=a^{-2} R_{\mathrm{pl}}(\beta+\phi, \lambda-\log a) .
$$

Equation (11) is a translation invariant description of the input signal, while the rotation and scaling have become translations along the new coordinate system axes. If we would perform a Fourier transform on $T_{\mathrm{pl}}(\lambda, \beta)$, this is called a Fourier-Mellin transform.

We will introduce a similar procedure as we showed from equations (7) to (11) that can be applied to minutiae sets in order to find a representation which is invariant to translation and where rotation and scaling are translations. 


\subsection{An analytical spectral minutiae representation}

When implementing the Fourier transform there are two important issues that should be considered. First, when a discrete Fourier transform is taken of a continuous image, this results in a description of a periodic repetition of the original image. This is undesirable because it introduces errors. Second, the re-mapping onto a polarlogarithmic coordinate system after using a discrete Fourier transform introduces interpolation artifacts. Therefore we introduce an analytical representation of the input minutiae, and then use analytical expressions of a continuous Fourier transform that are evaluated on every grid point in the polar-logarithmic plane. These analytical expressions are obtained as follows. Assume we have a fingerprint with $Z$ minutiae. With every minutia, a function $m_{i}(x, y)=\delta\left(x-x_{i}, y-y_{i}\right), i=1, \ldots, Z$ is associated where $\left(x_{i}, y_{i}\right)$ represents the location of the $i$-th minutia in the fingerprint image. Thus, in the spatial domain, every minutia is represented by a Dirac pulse. The Fourier transform of $m_{i}(x, y)$ is given by:

$$
\mathscr{F}\left\{m_{i}(x, y)\right\}=\exp \left(-\mathrm{j}\left(\omega_{\mathrm{x}} x_{i}+\omega_{\mathrm{y}} y_{i}\right)\right),
$$

and the spectral representation of the minutiae is defined as

$$
\mathcal{M}\left(\omega_{\mathrm{x}}, \omega_{\mathrm{y}}\right)=\sum_{i=1}^{Z} \exp \left(-\mathrm{j}\left(\omega_{\mathrm{x}} x_{i}+\omega_{\mathrm{y}} y_{i}\right)\right) .
$$

This is the analytical expression for the spectrum which can be directly evaluated on a polar-logarithmic grid. The resulting representation in the polar-logarithmic domain is invariant to translation, while rotation and scaling of the input have become translations along the polar-logarithmic coordinates.

\subsection{Implementation}

In order to obtain our final spectral representation, the continuous spectrum (13) is sampled on a polar-logarithmic grid. In the radial direction $\lambda$ we use $M=128$ samples logarithmically distributed between $\lambda=0.1$ and $\lambda=0.6$. In the angular direction $\beta$, we use $N=256$ samples uniformly distributed between $\beta=0$ and $\beta=\pi$. Because of the symmetry of the Fourier transform for real-valued functions, using the interval between 0 and $\pi$ is sufficient. This polarlogarithmic sampling process is illustrated in Figure 1.

The examples of the minutiae spectra are shown in Figure 2. For each spectrum, the horizontal axis represents the rotation angle of the spectral magnitude (from 0 to $\pi$ ); the vertical axis represents the frequency of the spectral magnitude (the frequency increases from top to bottom). We can notice that the minutiae spectrum is periodic on the horizontal axis.

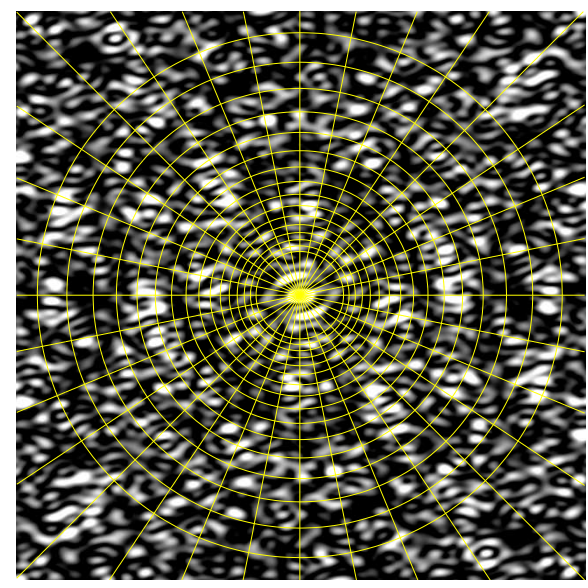

(a)

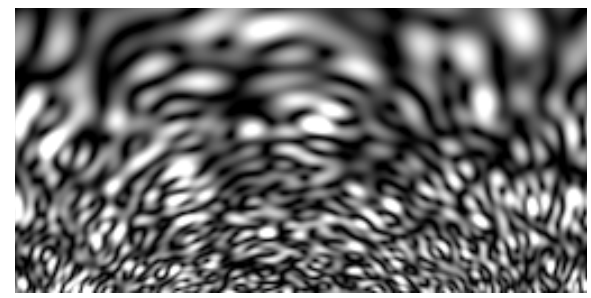

(b)

Figure 1. Illustration of the polar-logarithmic sampling. (a) the Fourier spectrum in a Cartesian coordinate and a polar-logarithmic sampling grid; (b) the Fourier spectrum sampled on a polarlogarithmic grid.

\section{Spectral Minutiae Matching}

After representing fingerprints in the form of minutiae spectra, the next step is matching: the comparison of two minutiae spectra. The result of matching is either a 'match' (the two spectra appear to be from the same finger) or a 'non-match' (the two spectra appear to be from different fingers). Normally, in this step, we will first compute a number (similarity score) which corresponds to the degree of similarity. Then, by using a threshold, we can make a match/non-match decision [6].

\subsection{Direct matching}

Let $R(m, n)$ and $T(m, n)$ be the two sampled minutiae spectra in the polar-logarithmic domain respectively achieved from the reference fingerprint and test fingerprint. Both $R(m, n)$ and $T(m, n)$ are normalized to have zero mean and unit energy. As a similarity score, the correlation of two minutiae spectra was chosen, which is a common similarity measure in image processing. Therefore, the matching score between $R$ and $T$ is defined as:

$$
S_{\mathrm{DM}}^{(R, T)}=\frac{1}{M N} \sum_{m, n} R(m, n) T(m, n) .
$$




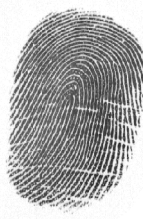

(a)

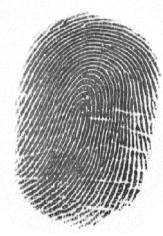

(c)

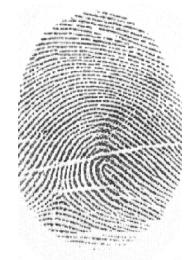

(e)

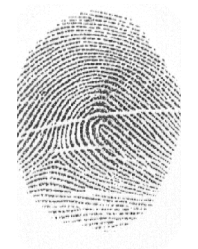

(g)

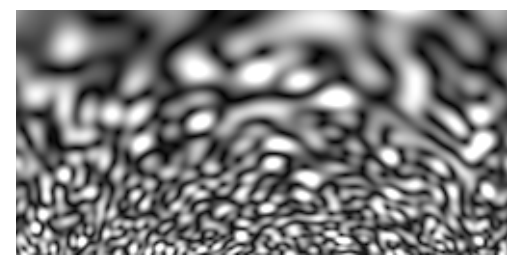

(b) Minutiae spectrum of (a)

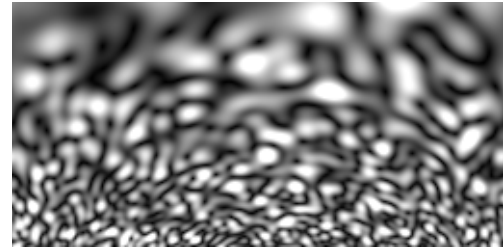

(d) Minutiae spectrum of (c)

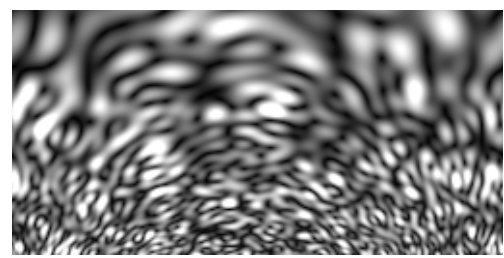

(f) Minutiae spectrum of (e)

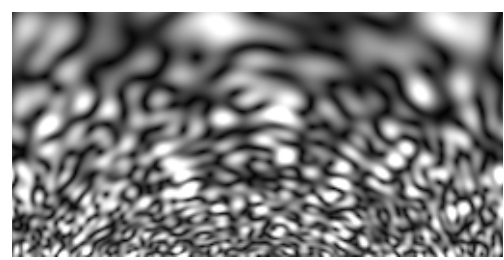

(h) Minutiae spectrum of $(\mathrm{g})$
Figure 2. Examples of minutiae spectra. (a) and (c) are fingerprints from the same finger; (e) and (g) are fingerprints from the same finger.

\subsection{Weighted sum correlation matching}

Let $R(m, n)$ and $T(m, n)$ be as defined in the previous subsection. The line correlation $C^{(R, T)}(m)$ of $R(m, n)$ and $T(m, n)$ is defined as

$$
C^{(R, T)}(m)=\frac{1}{N} \sum_{n=1}^{N} R(m, n) T(m, n),
$$

for $m=1 \ldots M$, where $M=128, N=256$.

During matching, a weighted sum rule for the line correlation values is chosen as the similarity score of $R(m, n)$ and $T(m, n)$, which is defined as:

$$
S_{\mathrm{WSC}}^{(R, T)}=\frac{1}{M} \sum_{m=1}^{M} w(m) C^{(R, T)}(m),
$$

with $w(m)$ the sum rule weight for the correlation value $C^{(R, T)}(m)$. The weights $w(m)$ need to be obtained by training. It is chosen as:

$$
w(m)=\frac{\mu_{G}(m)-\mu_{I}(m)}{\sqrt{\sigma_{G}(m) \sigma_{I}(m)}},
$$

which is related to the detection index used in communication theory [17]. In (17), $\mu_{G}(m)$ and $\sigma_{G}(m)$ are the mean and the standard deviation of $C^{(R, T)}(m)$ in case $R$ and $T$ are from the same finger (a genuine pair), and $\mu_{I}(m)$ and $\sigma_{I}(m)$ are the mean and the standard deviation of $C^{(R, T)}(m)$ in case $R$ and $T$ are from different fingers (an imposter pair).

\subsection{Fast rotation shift searching}

In most fingerprint databases, there is no scaling difference between the fingerprints, or the scaling can be compensated for on the level of the minutiae sets [4]. Therefore, in practice only rotations have to be compensated for. This is done by testing a few rotations. Because we applied the polar-logarithmic transform to the Fourier spectra, the rotation becomes the circular shift in the horizontal direction in our minutiae spectra. We chose to test rotation from $-10^{\circ}$ to $+10^{\circ}$, which corresponds circular shifts from -15 units to +15 units in the polar-logarithmic domain. This rotation range is fingerprint data dependent. If big rotations appeared often in fingerprint samples, then a larger rotation range should be applied. Let $T_{k}(m, n)$ be defined as $T(m, n)$ with a circular shift $k$ in the horizontal direction. For each shift trial, a new similarity score $S^{\left(R, T_{k}\right)}$ is calculated using (14) or (16). Finally, the highest score is chosen as the final matching score and the corresponding shift $k$ is recorded as the best shift (that is, the best rotation).

We applied a fast search for the best shift. This algorithm consists of the following steps:

(1) 5 circular shifts $(k=-12,-6,0,6,12)$ are applied to $T(m, n)$ and the similarity scores $S^{\left(R, T_{k}\right)}$ are calculated. The maximum value of $S^{\left(R, T_{k}\right)}$ is denoted as $S_{1}$ and its corresponding shift $k$ is denoted as $k_{1}$;

(2) 2 circular shifts $\left(k=k_{1}-2, k_{1}+2\right)$ are applied to $T(m, n)$, and the similarity scores $S^{\left(R, T_{k}\right)}$ are calculated. The maximum value of $S^{\left(R, T_{k}\right)}$ and $S_{1}$ is denoted as $S_{2}$, and its corresponding shift $k$ is denoted as $k_{2}$;

(3) 2 circular shifts $\left(k=k_{2}-1, k_{2}+1\right)$ are applied to $T(m, n)$, and the similarity scores $S^{\left(R, T_{k}\right)}$ are calculated. The maximum value of $S^{\left(R, T_{k}\right)}$ and $S_{2}$ is denoted as $S_{\text {final }}$.

Using this fast rotation shift search algorithm, only 9 shift trials need to be tested, instead of 31 shift trials for an exhaustive search. After these steps, the value $S_{\text {final }}$ is recorded as the final matching score between $R$ and $T$. We tested both fast search and exhaustive search methods, and gained similar results. But, theoretically, this fast search solution is heuristic and may not give optimal results. 


\section{Results}

\subsection{Measurements}

We test the spectral minutiae representation in a verification setting. A verification system authenticates a person's identity by comparing the captured biometric characteristic with her own biometric template(s) pre-stored in the system. It conducts a one-to-one comparison to determine whether the identity claimed by the individual is true [11].

The matching performance of a fingerprint verification system is evaluated by means of several measures. The most commonly used are the false acceptance rate (FAR), the false rejection rate (FRR), and the equal error rate (EER). FAR is the probability that the system gives a 'match' decision for fingerprints that are not from the same finger. FRR is the probability that the system gives a 'non-match' decision for fingerprints that are from the same finger. When the decision threshold of a biometric security system is set so that the FAR and FRR are equal, the common value of FAR and FRR is referred to as the EER. For simplicity, we use EER as a performance indicator of our scheme.

The proposed algorithms have been evaluated by applying them to the MCYT Biometric Database [12]. We used the fingerprint data containing 3600 fingerprints. They were obtained from the first 30 individuals (person ID from 0000 to 0029 in MCYT). Each individual contributed data from 10 different fingers, and from each finger, 12 samples were collected using the optical sensor U.are.U from Digital Persona [1], with a resolution of 500dpi. The minutiae sets were obtained by the VeriFinger minutiae extractor [3].

Among our fingerprint dataset, we used 1200 fingerprint samples from 10 individuals (person ID from 0020 to 0029 in MCYT) as a training set to calculate the weighted sum correlation weights (17), and 2400 fingerprint samples from 20 individuals (person ID from 0000 to 0019 ) as the test set.

For each comparison, we chose two fingerprints from the data set: one as a reference fingerprint, another one as a test fingerprint. For matching verification (genuine pairs), we used all the possible combinations, thus we have in total $10 \times 10 \times\left(\begin{array}{c}12 \\ 2\end{array}\right)=6600$ genuine scores in the training set, and $20 \times 10 \times\left(\begin{array}{c}12 \\ 2\end{array}\right)=13200$ genuine scores in the test set. For non-matching verification (imposter pairs), we compared each fingerprint with 10 randomly chosen samples from other individuals, thus we have in total $1200 \times 10=12000$ imposter scores in the training set, and $2400 \times 10=24000$ imposter scores in the test set.

The weights (17) for the weighted sum correlation matching that we obtained from the training set are shown in Figure 3. The EERs we achieved from the test dataset are shown in Table 1. The genuine and imposter distributions are shown in Figure 4. The FAR, FRR and ROC (Receiver Operating Characteristic) curves are shown in Figure 5 and 6 respectively. In these figures, the matching scores are nor-

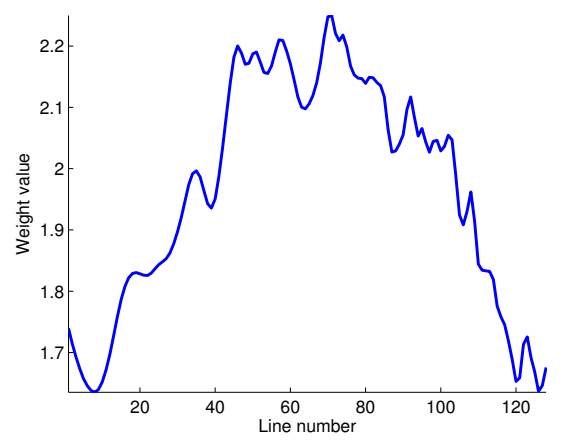

Figure 3. The weights for each line correlation.

Table 1. Matching results (the test dataset).

\begin{tabular}{|l|c|}
\hline Matching method & EER \\
\hline \hline Direct matching (DM) & $3.21 \%$ \\
\hline Weighted sum correlation (WSC) & $3.13 \%$ \\
\hline
\end{tabular}

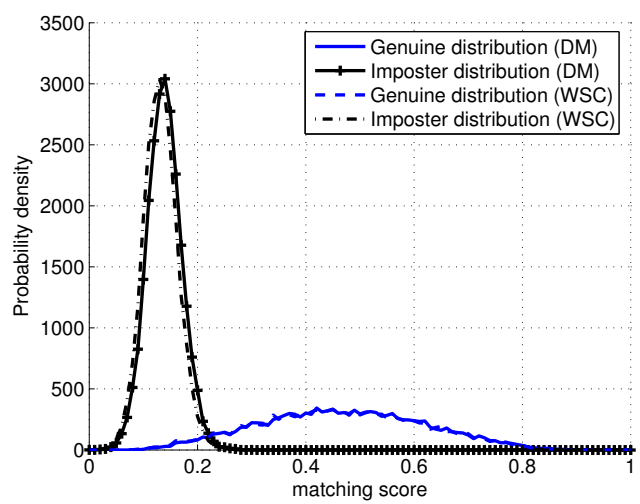

Figure 4. Genuine and imposter distributions (the test dataset).

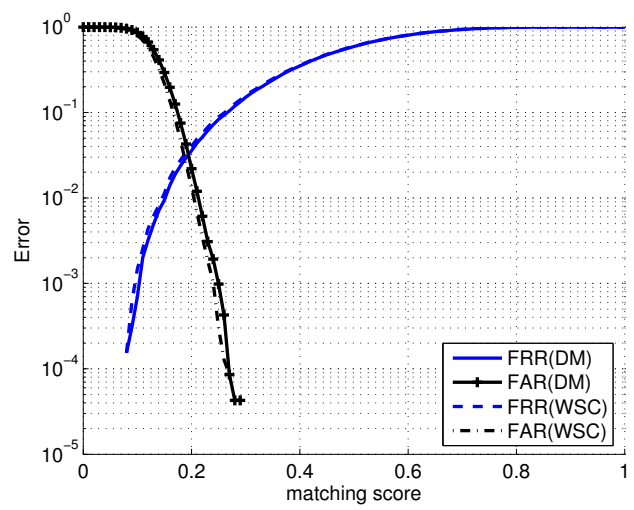

Figure 5. FAR and FRR curves (the test dataset).

malized to the interval $[0,1]$ for a better comparison.

From Table 1, we can see that the weighted sum correlation matching (WSC) received a small improvement compared with the direct matching (DM). From Figure 4, the genuine score distributions for the two matching algo- 


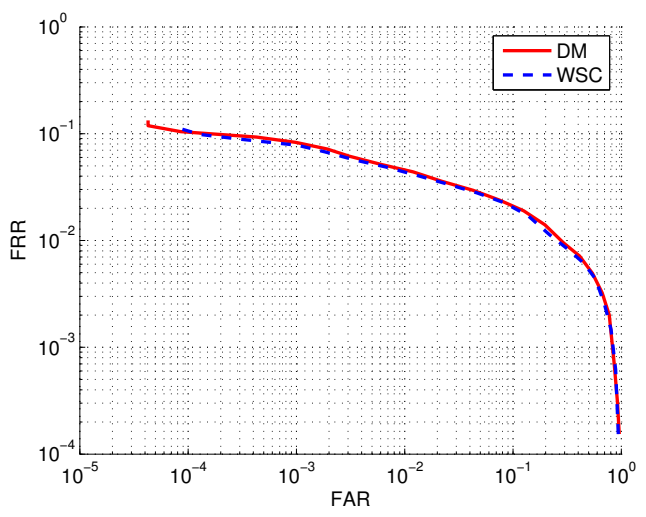

Figure 6. ROC curves (the test dataset).

rithms are almost overlapping, while the imposter scores from WSC are slightly lower. However, based on the very small difference in EERs, we cannot state that WSC is a better matching algorithm.

To evaluate our algorithm, we compared our results with the ones from other fingerprint recognition systems. From the academic domain, the MCYT organizer reports an EER with 5.5\% using minutiae-based algorithm [12]. From the commercial domain, we tested the performance of VeriFinger from Neurotechnologija, whose algorithm achieved one of the best results in both FVC2006 and FpVTE 2003 from NIST [3]. VeriFinger received a much better result with an EER $0.34 \%$. In our method, to combine with template protection schemes, we cannot perform an alignment between the reference and test minutiae sets, which is a crucial step for minutiae-based matching. This may cause the degradation of our algorithm. The comparison shows that although our result is acceptable for academic research, we still need to improve our algorithm to reach the security level of the current top fingerprint recognition systems.

\section{Conclusion}

The spectral minutiae representation is a new minutiaebased approach. Our method represents an unordered minutiae set as a fixed-length feature vector, which enables the combination of fingerprint recognition systems and template protection schemes. Moreover, this method avoids the minutiae registration difficulties by representing a minutiae set as a translation-invariant spectrum, in which the rotation and scaling become translations, so that they can be easily compensated for. In this paper, we also presented spectral minutiae matching algorithms and showed the experimental results. However, severe fingerprint non-linear distortions, noisy and missing minutiae can reduce the accuracy of our system. To make our method more robust to minutiae errors is our future work.

\section{Acknowledgment}

This research is supported by the research program Sentinels (http://www.sentinels.nl) and conducted in cooperation with Philips Research Laboratories.

\section{References}

[1] Digital Persona. http://www.digitalpersona.com/.

[2] United States Visitor and Immigrant Status Indicator Technology Program (US-VISIT). http://www.dhs.gov/us-visit.

[3] VeriFinger SDK. http://www.neurotechnologija.com/.

[4] ISO/IEC 19794-2, Information Technology - Biometric Data Interchange Format - Part 2: Finger Minutiae Data. 2005.

[5] A. Bazen and S. Gerez. Fingerprint matching by thin-plate spline modelling of elastic deformations. Pattern Recognition, 36(8):1859-1867, Aug. 2003.

[6] R. Bolle, J. H. Connell, S. Pankanti, N. K. Ratha, and A. W. Senior. Guide to Biometrics. Springer Verlag, 2003.

[7] D. Casasant and D. Psaltis. Position, rotation, and scale invariant optical correlation. Applied Optics, 15(7):17951799, July 1976.

[8] Q. Chen, M. Defrise, and F. Deconinck. Symmetric phaseonly matched filtering of fourier-mellin transform for image registration and recognition. IEEE Trans. PAMI, 16:11561168, 1994.

[9] A. Jain, L. Hong, and R. Bolle. On-line fingerprint verification. IEEE Trans. PAMI, 19(4):302-314, Apr. 1997.

[10] A. Jain, S. Prabhakar, L. Hong, and S. Pankanti. Filterbankbased fingerprint matching. IEEE Trans. Image Processing, 9(5):846-859, May 2000.

[11] D. Maltoni, D. Maio, A. Jain, and S. Prabhakar. Handbook of Fingerprint Recognition. Springer, New York, 2003.

[12] Ortega-Garcła, J., et al. MCYT baseline corpus: a bimodal biometric database. In IEE Proc. Vision, Image and Signal Processing 150(6), pages 395-401, 2003.

[13] C.-H. Park, M. J. T. Smith, M. Boutin, and J.-J. Lee. Fingerprint matching using the distribution of the pairwise distances between minutiae. In AVBPA, pages 693-701, 2005.

[14] Y. Sheng and J. Duvernoy. Circular-fourier-radial-mellin transform descriptors for pattern recognition. J. of the Optical Society of America A, 3(6):885-888, June 1986.

[15] P. Tuyls, A. Akkermans, T. Kevenaar, G. Schrijen, A. Bazen, and R. Veldhuis. Practical biometric authentication with template protection. In AVBPA, pages 436-446, 2005.

[16] P. Tuyls, B. Skoric, and Tom Kevenaar (Eds). Security with Noisy Data - On Private Biometrics, Secure Key Storage and Anti-Counterfeiting. Springer, 2007.

[17] H. van Trees. Detection, Estimation and Modulation Theory, Part I. John Wiley and Sons, New York, 1968.

[18] A. Willis and L. Myers. A cost-effective fingerprint recognition system for use with low-quality prints and damaged fingertips. Pattern Recognition, 34(2):255-270, 2001. 